\title{
Mode d'action des gènes et hétérosis pour le caractère montée à graines dans le croisement de deux lignées fixées de betterave à sucre (Beta vulgaris L.)
}

\author{
F. Le Cochec et P. Soreau \\ avec la collaboration technique de J.M. Retailleau
}

INRA, Station d'amélioration des plantes, Centre de recherches de Rennes, Domaine de la Motte au Vicomte, BP 29, 35650 Le Rheu, France

(reçu le 11 avril 1988, accepté le 20 mars 1989)

\begin{abstract}
Résumé - Les parents et les générations $F_{1}, F_{2}, B_{1}$ et $B_{2}$ issues du croisement de 2 lignées fixées de betterave à sucre, l'une sensible et l'autre résistante, ont été testés pour leur comportement vis-à-vis de la montée à graines en conditions de semis très précoce. Les générations sont fréquemment différentes pour ce caractère. Elles ont été analysées en utilisant une méthode des moindres carrés pondérée. La variation entre générations pour les montées précoces, moyennes et tardives est expliquée respectivement par un modèle à 4 paramètres (moyenne, additivité, dominance et additivité $x$ additivité), à 3 paramètres (moyenne, additivité et dominance) et à 2 paramètres (moyenne et additivité). La variation entre générations pour l'ensemble des montées est expliquée par un modèle à 3 paramètres (moyenne, additivité et dominance). En cas de dominance, les allèles responsables de la résistance sont plus fréquemment dominants que les allèles qui provoquent la sensibilité à la montée à graines. Une manifestation d'hétérosis (déviation de la $F_{1}$ par rapport à la moyenne des parents) due aux seuls effets de dominance a été observée pour les montées à graines totales. Une manifestation d'hétérosis due aux effets de dominance et d'épistasie a été observée pour les montées précoces.
\end{abstract}

betterave à sucre - lignées fixées - montée à graines - hétérosis - déterminisme génétique

\begin{abstract}
Summary - Gene action and heterosis for the bolting character in a cross between two sugar beet inbred lines (Beta vulgaris L.). Parents, $F_{1}, F_{2}$ and backcross generations of a cross between 2 higly inbred sugar beet lines, one susceptible and one resistant, were evaluated for bolting characteristics in early sowing conditions. Generation means were significantly different for many characteristics and were analyzed using a weighted least squares procedure. The variation among generation means for early, middle and late bolters was explained respectively by a 4parameter model (mean, additivity, dominance and additivity $x$ additivity), a 3-parameter model (mean, additivity and dominance) and a 2-parameter model (mean and additivity). A 3-parameter model (mean, addivitity and dominance) accounted for most of the variation among generations for whole bolters. In case of dominance, alleles responsible for resistance were dominant much more often than alleles responsible for susceptibility to bolting. Heterosis $\left(F_{1}\right.$ deviation from mid-parent) as a consequence of dominance effects was observed for whole bolters. Heterosis as a consequence of dominance and epistatic effects was observed for early bolters.
\end{abstract}

sugar beet - inbred lines - bolting - heterosis - gene effects

\section{Introduction}

La montée à graines chez la betterave correspond à une élongation de la tige sur des plantes qui étaient jusqu'alors au stade de rosette, et elle peut se manifester dès le début de l'été dans les semis de printemps. Elle marque le passage du stade végétatif au stade reproducteur, qui aboutit à la floraison, la fécondation et la formation des glomérules. L'initiation de la montaison et de l'induction florale résulte de l'interaction de facteurs dus au milieu et de facteurs génétiques. Elle nécessite d'abord une période de vernalisation à basse température puis une période d'éclairement avec une quantité et une qualité de lumière caractéristiques dans des conditions de tempéra- 
ture déterminées. Ces facteurs externes réagiraient avec des mécanismes physiologiques internes, pour favoriser la synthèse d'une hormone de floraison de nature encore inconnue (Lexander, 1980).

Certaines plantes ont besoin de moins de froid et des conditions d'éclairement et de température différentes des autres plantes. Elles peuvent, de ce fait, monter plus ou moins tôt en saison. $\mathrm{Ce}$ caractère est économiquement et agronomiquement très défavorable, car il correspond non seulement à une réduction de la production et de la teneur en sucre des racines ( $\mathrm{O}^{\prime} \mathrm{C}$ onnor et Fitzgerald, 1987), mais il provoque en outre des risques importants d'infestation du sol par les graines de plantes devenues annuelles (Longden et Goddard, 1987).

Abbegg (1936) a démontré que la betterave annuelle de Munerati comportait un gène dominant $B$ et des gènes complémentaires pouvant augmenter le taux des montées. II existe par ailleurs dans certaines betteraves cultivées un allèle $B^{\prime}$ différent de $B$, capable d'induire le caractère annuel (Owen et al., 1940). En fait, ces 2 allèles n'existent plus qu'à une faible fréquence dans le matériel commercialisé en Europe occidentale et l'hérédité des montées à graines que l'on constate actuellement est tout à fait différente et plus complexe que celle présentée par les premiers observateurs.

Marcum (1948) a montré qu'en semis précoce, les hybrides issus du croisement d'un parent mâle stérile sensible $(58,7 \%$ de montées) et d'un pollinisateur diploïde résistant $(1,6 \%$ de montées) montaient entre 28,5 et $2,7 \%$ ( $19,2 \%$ en moyenne), et il en a conclu que la résistance est un caractère dominant. Des résultats analogues ont été signalés par Bolelova et al. (1984), qui croisent 2 parents montant à 84,5 et $0 \%$ et obtiennent des hybrides montant entre 0 et $22 \%$. Desprez (1985), se basant sur une série d'observations de familles multigermes autostériles et de lignées monogermes autofertiles en cours de sélection massale, ainsi que sur le comportement d'hybrides trois-voies, en déduit que la sensibilité à la montée à graines est due à plusieurs gènes récessifs agissant différemment, certains étant plus sensibles à l'action des jours croissants et d'autres à l'action des basses températures.

La présente étude a été entreprise afin d'essayer de préciser le déterminisme des gènes qui conditionnent la montée à graines chez la betterave à sucre. On a estimé qu'en préalable à une telle étude, il était nécessaire de sélectionner des lignées fixées (ou homozygotes) bien différenciées pour leur comportement vis-à-vis de la montée à graines.

\section{Matériel et Méthodes}

A la suite de tests préliminaires effectués en 1984 et 1985 à la Station d'amélioration des plantes de Le Rheu, 2 lignées ont été retenues, dans une collection de lignées fixées de betterave à sucre, pour leur réaction à la montée à graines en semis très précoce au printemps : une lignée MGLB (dénommée $P_{1}$ ), très sensible, qui avait monté à $90 \%$ dans un semis du 9 février, et une lignée 569-43 (dénommée $P_{2}$ ), très résistante à la montée dans des conditions analogues ( $0 \%$ dans un semis du 20 février).

Ces 2 lignées ont un taux d'homozygotie très élevé. MGLB, lignée pseudocompatible provenant d'une famille d'origine française, a été autofécondée pendant 12 générations et 569-43, lignée autofertile d'origine américaine, pendant 10 générations successives. Cette dernière lignée est un type $O$ (mainteneur de stérilité), monogerme, sélectionné dans la souche USDA C-569 reçue en 1969 de la station de Salinas. Elle a été recroisée chaque année sur le parent mâle stérile $569-\mathrm{HO}-43$, de sorte que le type $\mathrm{O}$ et la lignée mâle stérile présentent un aspect identique et un comportement similaire, pour la montée à graines en particulier.

Les parents $P_{1}$ et $P_{2}$ et les générations $F_{1}$, mâle stérile et mâle fertile, $F_{2}$ issue de la multiplication de la génération mâle fertile $F_{1}, B_{1}$ et $B_{2}$ provenant respectivement du croisement des plantes $F_{1}$ mâle stériles par les parents $P_{1}$ et $P_{2}$, ont été utilisés dans cette expérimentation.

Les plantes $F_{1}$ mâle fertiles ont été reconnues dans le croisement des 2 parents à l'aide du gène marqueur dominant $R$, provenant de la lignée $569-43$, qui provoque la couleur rouge de l'hypocotyle. La lignée MGLB se comporte comme un type $O$ en croisement avec la lignée mâle stérile $569-\mathrm{HO}-43$, de sorte que leur hybride mâle stérile a pu être utlisé pour obtenir les générations $B_{1}$ et $B_{2}$. Les générations $F_{1}$ mâle stérile et $F_{1}$ mâle fertile ont été récoltées en 1985 sous des isoloirs de popeline, et les générations $P_{1}, P_{2}, F_{1}$ mâle stérile, $F_{2}, B_{1}$ et $B_{2}$, en 1986 dans des isolements par la distance mis en place en différents endroits à proximité de la station.

En 1987, les 6 générations ont été semées le 13 février dans un essai en blocs randomisés à 6 répétitions à l'intérieur d'un tunnel non chauffé couvert de bâches transparentes. En raison des grandes différences de variabilité génétique entre les générations et pour mieux estimer le pourcentage des montées à graines dans les générations en ségrégation, chaque répétition comportait le nombre suivant de lignes par génération : $P_{1}, P_{2}$ et $F_{1}$, une ligne, $B_{1}$ et $B_{2}, 2$ lignes et $F_{2}, 3$ lignes. Les lignes d'une longueur de $3,60 \mathrm{~m}$ étaient séparées entre elles de $0,30 \mathrm{~m}$. Le tunnel a été découvert le 31 mars, peu après l'apparition des 2 premières feuilles vraies. Un démariage à $4 \mathrm{~cm}$ entre les plantes a été réalisé le 14 avril. Le nombre de plantes par ligne a varié de 58 à 71 plantes suivant les générations. Les températures moyennes au niveau du sol ont été de $6^{\circ} 6$ en février, $7^{\circ} 9$ en mars et $9^{\circ} 4$ en avril. Par la suite, la culture a été l'objet des traitements herbicides, aphicides et fongicides normaux.

Les plantes montées ont été dénombrées et arrachées dès qu'elles devenaient visibles au-dessus du niveau moyen des feuilles. Cette opération a débuté le 25 juin et a été renouvelée tous les 5 jours jusqu'au 21 octobre. Les plantes demeurant en place le 21 octobre ont été considérées comme non montées. 
La période comprise entre le 25 juin et le 21 octobre a été divisée en 3 périodes à compter du 25 juin, les montées précoces correspondant à celles apparues entre le 25 juin et le 25 juillet, les montées moyennes entre le 25 juillet et le 25 août et les montées tardives après le 25 août. Sur un total de 3833 plantes dénombrées dans l'essai, 159 plantes ont été classées dans les montées précoces, 276 plantes dans les montées moyennes et 171 plantes dans les montées tardives.

\section{Méthodes statistiques}

Les calculs ont été réalisés à partir des pourcentages de montées de chaque parcelle. En raison des variations importantes des pourcentages entre générations, les données ont subi avant analyse une transformation angulaire et les résultats sont présentés en valeurs transformées.

L'analyse de la variance a permis de tester la signification des différences entre les générations et entre les répétitions. II n'existe pas de différences significatives entre les répétitions pour les 4 classes de montées. On a donc considéré que la meilleure estimation de la variance de la moyenne de chaque génération et de l'hétérosis était obtenue en divisant la variance entre répétitions par le nombre de répétitions.

Pour les 4 classes de montées, les valeurs moyennes de chaque génération ont été utilisées pour connaître le mode d'action des gènes conditionnant la montée à graines. Elles ont été comparées à l'aide d'un test $\chi^{2}$ aux valeurs obtenues dans un modèle à 2 paramètres (moyenne $m$, additivité $d$ ). Si le test est significatif, elles ont été comparées aux valeurs obtenues dans un modèle à 3 paramètres (moyenne $m$, additivité $d$, dominance $h$ ) puis éventuellement dans un modèle à 4 paramètres (moyenne $m$, additivité $d$, dominance $h$, additivité $\mathrm{x}$ additivité $i$ ), jusqu'à l'obtention d'une valeur non significative du test $\chi^{2}$, indiquant une bonne adéquation du modèle aux données.

Les valeurs des générations peuvent être exprimées de la façon indiquée dans le Tableau I, en fonction des effets $m, d, h$ et $i$.

$m, d, h$ et $i$ ainsi que leurs écarts types ont été calculés à partir de ces équations, qui décrivent un modèle linéaire de régression multiple. La distribution du $\chi^{2}$ est utilisée pour faire un test sur le modèle en utilisant une mesure des fluctuations aléatoires issues des répétitions. Les statistiques de $\chi^{2}$ ont été calculées en pondérant les écarts entre les valeurs observées et théoriques des moyennes des générations par l'inverse de leur variance (Mather et Jinks, 1977).

La distribution du $\chi^{2}$ comporte 4 degrés de liberté pour le modèle à 2 paramètres, 3 degrés de liberté pour le modèle à 3 paramètres et 2 degrés de liberté pour le modèle à 4 paramètres. Toutefois, pour les montées précoces, le nombre des degrés de liberté a été réduit de une unité pour chaque modèle, la valeur nulle de la génération $P_{2}$ n'ayant pas permis de réaliser une pondération de la valeur de cette génération.

L'homogénéité des variances résiduelles associées à chaque génération a été vérifiée dans chaque cas, pour les variances non nulles, à l'aide du test de Bartlett.

L'adaptation du modèle à 3 paramètres aux valeurs observées a également été vérifiée à l'aide des 3 tests individuels $A, B$ et $C$ d'additivité et de dominance des effets géniques. En l'absence d'épistasie, les différences : $B_{1}-1 / 2\left(P_{1}+F_{1}\right), B_{2}-1 / 2\left(P_{2}+F_{1}\right)$ et $F_{2}-1 / 4\left(2 F_{1}+P_{1}+P_{2}\right)$ sont nulles. II faut bien voir cependant que les $3 \chi^{2}$ à un degré de liberté implicitement utilisés dans ces tests ne sont pas indépendants et qu'il s'agit donc seulement d'une vérification supplémentaire du test $\chi^{2}$ précédent. La signification de la valeur de ces 3 tests individuels a été calculée en utilisant un test $t$. mule:

Les valeurs d'hétérosis ont été estimées par la for-

$$
F_{1}-1 / 2\left(P_{1}+P_{2}\right)
$$

et les valeurs relatives d'hétérosis par la formule :

$$
\frac{F_{1}-1 / 2\left(P_{1}+P_{2}\right)}{1 / 2\left(P_{1}+P_{2}\right)} \times 100
$$

Pour éviter les valeurs négatives, elles ont été calculées pour la résistance et non plus pour la sensibilité à la montée à graines.

\section{Résultats}

Les pourcentages de montée avant et après transformation angulaire pour chaque génération et pour chaque type de montée sont indiqués dans le Tableau II. Ils sont fréquemment différents ou très différents, dans les montées précoces et totales en particulier. Le parent sensible $P_{1}$ présente un nombre équivalent de montées précoces et moyennes, environ 3 fois supérieur à

Tableau 1. Valeurs des générations des 4 classes de montées en fonction des effets $m, d, h$ et $i$.

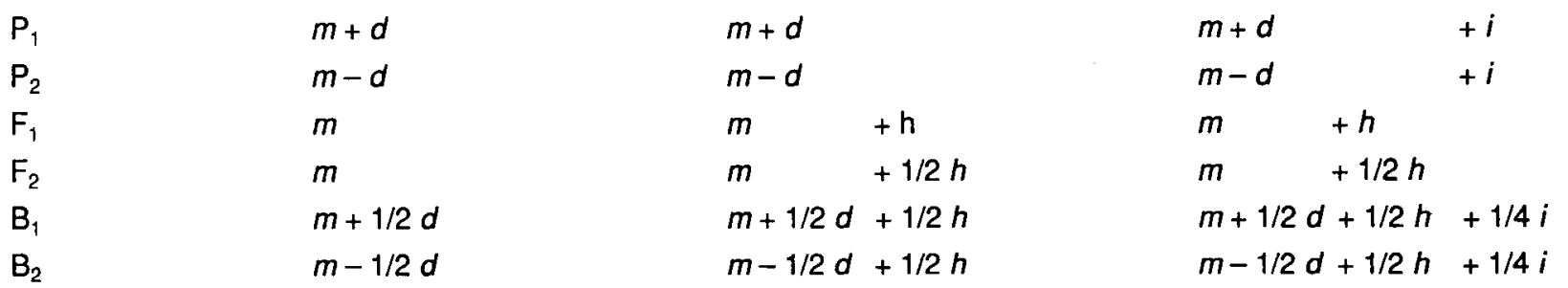


celui des montées tardives. Le parent résistant $\mathrm{P}_{2}$ n'a pas de montées précoces et très peu de montées moyennes et tardives.

La répartition relative des montées entre les générations apparaît différente suivant les 3 classes. Pour les montées tardives, elle correspond à peu près à celle provoquée seulement par des gènes additifs, alors que pour les montées précoces, moyennes et totales, on note une déviation systématique des générations $F_{1}, F_{2}, B_{1}$ et $B_{2}$ vers le parent résistant $P_{2}$, ce qui indique une dominance partielle mais prononcée du caractère résistance sur le caractère sensibilité à la montée à graines. Cette dominance est confirmée par la supériorité des $F_{2}$ sur les $F_{1}$.

Le test $\chi^{2}$ d'adéquation des modèles aux pourcentages de montée observés (Tableau III) montre que les modèles à 2 puis à 3 paramètres ne conviennent pas pour les montées précoces. Le test individuel $\mathrm{C}$ très différent de zéro confirme que le modèle à 3 paramètres n'est pas adapté. Cette classe de montées est expliquée par un modèle à 4 paramètres, le $\chi^{2}$ testant la valeur de l'ajustement étant loin du seuil de signification. En cas d'utilisation du test $t$, le paramètre $i$ apparaît néanmoins à la limite de la signification.

Les montées moyennes ne peuvent être expliquées par un modèle à 2 paramètres. Le modèle à 3 paramètres, bien que peu éloigné de la limite de la signification, convient et cela est confirmé par les valeurs nulles des 3 tests individuels.

Comme cela a été constaté plus haut, les valeurs observées dans les montées tardives peuvent être expliquées par un simple modèle

Tableau II. Moyenne des générations (valeurs transformées $t$ et valeurs non transformées n.t.) pour le pourcentage des montées à graines précoces, moyennes, tardives et totales dans un croisement de 2 lignées de betterave à sucre : MGLB $\left(P_{1}\right)$ et 569-43 $\left(P_{2}\right)$.

\begin{tabular}{|c|c|c|c|c|c|c|c|c|}
\hline \multirow[t]{2}{*}{ Générations } & \multicolumn{2}{|c|}{$\begin{array}{c}\text { Montées précoces } \\
\%\end{array}$} & \multicolumn{2}{|c|}{$\begin{array}{c}\text { Montées moyennes } \\
\%\end{array}$} & \multicolumn{2}{|c|}{$\begin{array}{c}\text { Montées tardives } \\
\%\end{array}$} & \multicolumn{2}{|c|}{$\begin{array}{c}\text { Montées totales } \\
\%\end{array}$} \\
\hline & $t$ & n.t & $t$ & $n . t$ & $t$ & $n . t$ & $t$ & n.t \\
\hline$P_{1}$ & 27,21 & 20,57 & 26,70 & 21,14 & 15,94 & 7,71 & 44,81 & 49,43 \\
\hline$P_{2}$ & 0 & 0 & 4,91 & 1,29 & 4,45 & 1,29 & 9,36 & 2,58 \\
\hline$F_{1}$ & 1,10 & 0,23 & 10,78 & 3,72 & 10,17 & 3,26 & 15,68 & 7,21 \\
\hline $\mathrm{F}_{2}$ & 10,36 & 3,23 & 14,37 & 6,23 & 11,14 & 3,88 & 21,44 & 13,34 \\
\hline $\mathrm{B}_{1}$ & 13,58 & 5,64 & 20,92 & 12,69 & 15,96 & 7,95 & 30,96 & 26,28 \\
\hline $\mathrm{B}_{2}$ & 1,19 & 0,25 & 4,34 & 0,87 & 7,95 & 2,12 & 9,98 & 3,24 \\
\hline ppds 0,05 & 3,11 & & 5,27 & & 3,53 & & 2,24 & \\
\hline
\end{tabular}

Tableau III. Paramètres génétiques et adaptation des modèles à 2, 3 et 4 paramètres aux pourcentages de montées à graines observés dans le croisement de 2 lignées de betterave à sucre : MGLB et 569-43 (valeurs transformées).

\begin{tabular}{|c|c|c|c|c|c|c|c|c|}
\hline \multirow{2}{*}{$\begin{array}{l}\text { Paramètres } \\
\text { génétiques } \\
\text { et test }\end{array}$} & \multicolumn{3}{|c|}{$\begin{array}{c}\text { Montées précoces } \\
(\%)\end{array}$} & \multicolumn{2}{|c|}{$\begin{array}{l}\text { Montées moyennes } \\
\text { (\%) }\end{array}$} & \multirow{2}{*}{$\begin{array}{c}\text { Montées tardives } \\
(\%) \\
2 \\
\text { paramètres }\end{array}$} & \multicolumn{2}{|c|}{$\begin{array}{c}\text { Montées totales } \\
(\%)\end{array}$} \\
\hline & $\begin{array}{c}2 \\
\text { paramètres }\end{array}$ & $\begin{array}{c}3 \\
\text { paramètres }\end{array}$ & $\begin{array}{c}4 \\
\text { paramètres }\end{array}$ & $\begin{array}{c}2 \\
\text { paramètres }\end{array}$ & $\begin{array}{c}3 \\
\text { paramètres }\end{array}$ & & $\begin{array}{c}2 \\
\text { paramètres }\end{array}$ & $\begin{array}{c}3 \\
\text { paramètres }\end{array}$ \\
\hline$m$ & $7,84 \pm 0,48$ & $16,51 \pm 1,31$ & $19,35 \pm 1,79$ & $13,74 \pm 0,55$ & $16,10 \pm 1,30$ & $10,85 \pm 0,44$ & $24,06 \pm 0,29$ & $27,14 \pm 0,38$ \\
\hline$d$ & $15,64 \pm 1,27$ & $11,64 \pm 1,39$ & $13.40 \pm 1,58$ & $13,06 \pm 1,23$ & $13,08 \pm 1,23$ & $6,06 \pm 0,91$ & $16,65 \pm 0,36$ & $17,96 \pm 0,38$ \\
\hline$h$ & & $-15,39 \pm 2,12$ & $-18,65 \pm 2,42$ & & $-4,91 \pm 1,86$ & & & $-11,94 \pm 0,95$ \\
\hline$i$ & & & $-6,65 \pm 2,53$ & & & & & \\
\hline A & & $-1,15 \pm 3,24$ & & & $4,36 \pm 4,05$ & & & $1,42 \pm 2,26$ \\
\hline$B$ & & $1,28 \pm 2,63$ & & & $-7,01 \pm 4,10$ & & & $-5,08 \pm 2,31$ \\
\hline C & & $12,03 \pm 4,03$ & & & $4,31 \pm 5,91$ & & & $0,21 \pm 3,55$ \\
\hline & $58,01 \mathrm{HS}$ & $8,06 \mathrm{~S}$ & $2,36 \mathrm{NS}$ & $24,55 \mathrm{HS}$ & $6,89 \mathrm{NS}$ & $3,15 \mathrm{NS}$ & $165,8 \mathrm{HS}$ & $6,64 \mathrm{NS}$ \\
\hline Probabilité & $P<0,005$ & $0,010<P<0,025$ & $0,10<P<0,25$ & $P<0,005$ & $0,05<P<0,10$ & $0,25<P<0,50$ & $P<0,005$ & $0,05<P<0,10$ \\
\hline
\end{tabular}

S, HS : signification aux niveaux de probabilité 0,05 et 0,01 respectivement; NS : non-signification au niveau de probabilité 0,05 . 
additivité, le test $\chi^{2}$ étant non significatif.

En ce qui concerne les montées totales, le modèle à 2 paramètres ne convient pas, alors que le modèle à 3 paramètres apparaît adapté (confirmé par les valeurs nulles des 3 tests individuels). Le paramètre $d$ est positif puisque le parent $P_{1}$ est le parent le plus sensible à la montée. Le signe négatif de $h$ est en relation avec la différence $F_{1}-1 / 2\left(P_{1}+P_{2}\right)$ et indique que le parent $P_{2}$ contribue à la dominance. Cela signifie aussi que les allèles qui provoquent la résistance sont beaucoup plus souvent dominants que ceux qui provoquent la sensibilité à la montée à graines. Dans l'ensemble, les gènes produisent leur action indépendamment les uns des autres et il n'y a pas d'interactions épistatiques entre eux.

En raison de l'homogénéité des variances, le caractère montées totales n'apparaît pas comme un caractère indépendant des montées précoces, moyennes et tardives mais comme la résultante de leur action. L'adéquation finale au modèle à 3 paramètres peut être expliquée par le fait que 159 plantes ont conduit à un modèle à 4 paramètres, 276 plantes à un modèle à 3 paramètres et 171 plantes à un modèle à 2 paramètres.

L'effet d'hétérosis pour la résistance à la montée est très significatif pour les montées précoces et totales (Tableau IV). II est peu important pour les montées moyennes et n'apparaît pas dans les montées tardives. L'hétérosis relatif est également le plus important pour les montées totales et précoces. Cet hétérosis est peut être une conséquence de l'origine géographique très différente des 2 parents.

\section{Discussion}

Moll et Stuber (1974) indiquent qu'il y a 3 causes génétiques possibles pour l'hétérosis : la superdominance, la dominance partielle ou complète et l'épistasie. Dans le cas présent où il n'y a pas superdominance, on peut donc conclure que l'hétérosis observé pour les montées précoces et totales est une conséquence des effets de domi- nance partielle et d'épistasie. On observe en effet un hétérosis faible ou nul pour les montées moyennes et pour les montées tardives où ces effets sont réduits ou nuls, alors que l'hétérosis est élevé pour les montées précoces en présence des effets de dominance et d'épistasie et pour les montées totales en présence des effets de dominance. Pour le caractère montée à graines, I'hétérosis se manifeste donc surtout au stade jeune.

L'importance des effets d'additivité indique qu'il y a une possibilité effective d'obtenir, par autofécondation et sélection à chaque génération, des génotypes très résistants à la montée à graines dans la descendance du croisement entre une lignée sensible et une lignée résistante.

Les résultats cités ont été obtenus dans un seul lieu avec 2 lignées et ils devraient donc être confirmés par des expérimentations analogues. Ils correspondent néanmoins aux observations des précédents auteurs sur la dominance partielle de la résistance et l'hérédité polygénique de ce caractère.

II ressort aussi de cette étude une simplification progressive du mode d'action des gènes à mesure que la saison s'avance et que la plante vieillit : les effets d'épistasie ne se retrouvent pas dans les montées moyennes et tardives, ni les effets de dominance dans les montées tardives.

Ces résultats vont dans le sens des conclusions de 2 précédentes études sur l'évolution des composantes de la variance génétique en fonction de l'âge de la plante. Kremer (1981) constate dans le cumul des accroissements de hauteur du pin maritime entre la cinquième et la douzième année une réduction de la variance de dominance et une augmentation de l'héritabilité au sens strict. De même, Hébert (1986) a montré chez le maïs une augmentation de l'héritabilité au sens strict de la vitesse d'apparition des feuilles à partir de la dixième feuille, c'est-à-dire à partir du 26 juin pour un semis du 24 avril.

Cette variation du déterminisme génétique en cours de croissance est une conséquence de la complexité des mécanismes physiologiques qui

Tableau IV. Hétérosis et hétérosis relatif pour le pourcentage des plantes résistantes à la montée à graines dans un croisement de 2 lignées de betterave à sucre : MGLB et 569-43 (données transformées).

\section{Montées précoces Montées moyennes Montées tardives Montées totales}

$\begin{array}{lcccc}\text { Hétérosis } & 12,50 \pm 1,43 & 5,02 \pm 2,45 & -0,02 \pm 1,30 & 11,41 \pm 1,06 \\ \text { Hétérosis relatif } & 14,54 \% & 6,07 \% & 0,08 \% & 15,65 \%\end{array}$


contrôlent l'induction florale et, en particulier, de leur sensibilité à l'action des facteurs du milieu climatique (température, éclairement, longueur du jour) (Arnold, 1982). Elle explique la difficulté que l'on peut rencontrer à réduire ou à supprimer les montées en pratiquant la sélection massale dans les familles. II est relativement simple dans un premier temps de sélectionner pour ce caractère quand il $\mathrm{y}$ a seulement effet additif des gènes. Il devient par la suite de plus en plus difficile de contrôler les montées qui sont en relation avec des effets de dominance et d'épistasie.

\section{Remerciements}

Les auteurs remercient vivement $M$. Lefort-Buson, $G$. Douaire et $C$. Brian des remarques faites à la lecture du manuscrit de cet article.

\section{Références}

Abegg F.A. (1936) A genetic factor for the annual habit in beets and linkage relationship. J. Agric. Res. 53, 493-511

Arnold M.H. (1982) The evolution of weed beet and the prevention of contamination. $45^{\text {th }}$ Winter Congress, inst. Int. Rech. Betterav., Bruxelles, pp. 7-14

Bolelova Z.A., Tikhonova V.G. \& Leshchenko E.V. (1984) Genetical and physiological aspects of bolting in sugar beet plants. Sel'sk. Khoz. Biologiya 10, 90-95 (Plant Breeding Abstracts, 1986, 1221)
Desprez M. (1985) Réflexion sur la montée à graine chez la betterave. Conférence prononcée pour le centenaire des Ets Janacsz à Chodow-Konin, 25 juin 1985, Ets. F. Desprez, BP 41, F. 59242 Templeuve $11 \mathrm{pp}$.

Hebert Y. (1986) Expression de la variabilité génétique du phénomène d'hétérosis au cours de la vie de la plante chez le maïs. Thèse Ecole Nationale Supérieure Agronomique de Rennes $141 \mathrm{pp}$.

Kremer A. (1981) Déterminisme génétique de la croissance en hauteur du pin maritime (Pinus pinaster Ait). III Evolution des composantes de la variance phénotypique et génotypique. Ann. Sci. For. (Paris) 38(3), 355375

Lexander K. (1980) Present knowledge of sugar bolting mechanisms. 43rd Winter Congress, Inst. Int. Rech. Betterav., Bruxelles, pp. 245-258

Longden P. \& Goddard V. (1987) Effects of weed beet on crop yield and processability. Br. Sugar Beet Rev. 55, 10-11

Marcum W.B. (1948) Inheritance of bolting resistance. Proc. Am. Soc. Sugar Beet Technol. 5, 154-155

Mather K. \& Jinks J.L. (1977) Introduction to Biometrical Genetics. Chapman and Hall, London $231 \mathrm{pp}$

Moll R.H. \& Stuber C.W. (1974) Quantitative genetics. Empirical results relevant to plant breeding. In: Advances in Agronomy (Brady N.C., ed.). Academic Press, New York, pp. 277-313

O'Connor L. \& Fitzgerald P. (1987) Out-of-season sugar beet searching for bolting-resistant varieties. Farm and Food Research AFT, Ireland, Feb. 1987, pp. 27-29

Owen F.V., Carsner E. \& Stout M. (1940) Photothermal induction of flowering in sugar beets. J. Agric. Res. 61, 101-124 